\title{
Update on field use of the available drugs for the chemotherapy of human African trypanosomiasis
}

\author{
P. P. SIMARRO ${ }^{1}$, J. FRANCO ${ }^{1}$, A. DIARRA ${ }^{2}$, J. A. RUIZ POSTIGO ${ }^{3}$ and J. JANNIN ${ }^{1}$ \\ ${ }^{1}$ World Health Organization, HTM/NTD 20, avenue Appia 1211, Geneva 27, Switzerland \\ ${ }^{2}$ World Health Organization, IST/CA, P.O. Box 820, Libreville, Gabon \\ ${ }^{3}$ World Health Organization, CTD/DCD, P.O. Box3: 7608, Nasr City, Cairo 11371, Egypt
}

(Received 27 October 2011; revised 9 December 2011 and 4 Fanuary 2012; accepted 5 Fanuary 2012; first published online 6 February 2012)

SUMMARY

Despite the fact that eflornithine was considered as the safer drug to treat human African trypanosomiasis (HAT) and has been freely available since 2001, the difficulties in logistics and cost burden associated with this drug meant that the toxic melarsoprol remained the drug of choice. The World Health Organization responded to the situation by designing a medical kit containing all the materials needed to use eflornithine, and by implementing a training and drugs distribution programme which has allowed a transition to this much safer treatment. The introduction of the combination of nifurtimox and eflornithine (NECT) has accelerated the shift from melarsoprol to the best treatment available, due to reduced dosage and treatment time for eflornithine that has significantly lessened the cost and improved the burden of logistics encountered during treatment and distribution. The decrease in the use of more dangerous but cheaper melarsoprol has meant a rise in the per patient cost of treating HAT. Although NECT is cheaper than eflornithine monotherapy, an unexpected consequence has been a continuing rise in the per patient cost of treating HAT. The ethical decision of shifting to the best available treatment imposes a financial burden on HAT control programmes that might render long-term application unsustainable. These factors call for continuing research to provide new safer and more effective drugs that are simple to administer and cheaper when compared to current drugs.

Key words: African trypanosomiasis, melarsoprol, eflornithine, nifurtimox, treatment combinations.

\section{INTRODUCTION}

By the end of the last century a growing number of cases of human African trypanosomiasis (HAT) were not responding to the old and far from ideal drugs available to treat HAT (Legros et al. 1999). Moreover, the production of some drugs was threatened, either by increasing price (pentamidine), halted production (eflornithine) or planned cessation of production (nifurtimox, suramin and melarsoprol) (Van Nieuwenhove, 2000). No new drugs were in the pipeline.

This review gives a comprehensive summary of the process followed to ensure availability of drugs for the treatment of HAT and the efforts to ensure the access of patients to the best treatment. The financial burden of such ethical decision is discussed.

\section{ACCESS TO TREATMENT}

The World Health Organization created in 1999 a network to ensure availability and affordability of anti-trypanosomal drugs and started a joint advocacy

* Corresponding author: HTM/NTD, 20, avenue Appia 1211 Geneva 27, Switzerland. Tel: +4122 791 1345. Fax: +41227914777. E-mail: simarrop@who.int campaign with the newly established Campaign for Access to Essential Medicines and Médecins Sans Frontières (MSF) to secure and maintain trypanocidal drug production. As a result, in 2001, the two manufacturers Aventis and Bayer committed not only to ensure manufacture but also to donate the drugs to the World Health Organization for free distribution.

However, whilst discussing the chemotherapy of HAT throughout the first years of the 21 st century, several commentators have complained about the primacy of the use of melarsoprol to treat secondstage gambiense HAT. The major issue being melarsorpol's unacceptable toxicity whilst the less toxic, albeit cumbersome to administer, eflornithine has been available for over 20 years (Chappuis et al. 2005; Balasegaram et al. 2006; Robays et al. 2008; Priotto et al. 2008).

Indeed, the choice of drug to treat HAT depends on the subspecies of trypanosome and the stage of the disease, as judged by results of a diagnostic lumbar puncture to determine whether parasites have become manifest within the central nervous system. However, the logistics and financial capacity of the national sleeping sickness control programmes (NSSCPs) and the technical level of staff responsible 
for administering treatment also impact greatly on local drug policy. Therefore, even though eflornithine, considered as the safer drug to treat HAT, was made freely available in 2001, the difficulties in logistics (each patient requiring huge doses of drug delivered in 56 infusions over 14 days in 14 litres of sterile saline) meant that melarsoprol remained the drug of choice in spite of its toxicity: a 5\% drug-induced death rate through reactive encephalopathy (Blum et al. 2001; Seixas, 2004; Burri, 2010). The fact that treatment failures had reached levels of $30 \%$ in some foci in Angola, the Democratic Republic of the Congo, South of Sudan and Uganda (Moore, 2005) compounded the shortcomings of melarsoprol as a drug. The World Health Organization responded to the situation by designing a medical kit containing all the materials needed to use eflornithine in a logistically simplified fashion. Furthermore the organization supported a vigorous programme of capacity building and drug distribution to ensure that HAT victims had access to the best treatment available.

However, the implementation of eflornithine as first-line treatment was accompanied by a number of concerns. The drug is trypanostatic, it has a short half-life in the body and the difficult administration schedule imposes the risk of insufficient compliance to the full treatment course. Furthermore, eflornithine resistance is easily selected in the laboratory (Vincent et al. 2010). Then the risk of inducing resistance to eflornithine in the field, in addition to existing melarsoprol resistance, could lead to the emergence of untreatable second-stage HAT. It therefore became essential to determine whether combination therapies might improve efficacy and reduce the risk of resistance (Moore, 2005).

Clinical trials were conducted to assess efficacy of combinations of eflornithine, melarsoprol and nifurtimox, a drug labelled to be used in treating Chagas' disease, with some reported efficacy in second-stage HAT (Janssens and De Muynck, 1977; Moens et al. 1984; Pepin et al. 1992). In each case combinations yielded improved efficacy although all combinations including melarsoprol retained unacceptable drug toxicity (Priotto et al. 2006; Bisser et al. 2007; Checchi et al. 2007).

Based on the preliminary data obtained during these clinical trials, a multicentre, randomized, noninferiority clinical trial comparing eflornithine monotherapy with the combination of nifurtimox and eflornithine (NECT) was conducted between 2003 and 2008. The study concluded that the combination of nifurtimox and eflornithine had a comparable safety and efficacy with eflornithine monotherapy. Furthermore, the combination allowed for reduced dose and treatment time for eflornithine which significantly lessened the cost and significantly improved the logistical burden for treatment and distribution (Priotto et al. 2009).
These positive results led to the WHO Expert Committee on the Selection and Use of Essential Medicines (held in March 2009) to recommend the inclusion of nifurtimox in the Model List of Essential Medicines (EML) to be used in combination with eflornithine for the treatment of the second-stage of Trypanosoma brucei gambiense infection (World Health Organization, 2009).

Following this inclusion, NEC'T was proposed as the best available therapeutic option to treat the second-stage of gambiense HAT. The World Health Organization secured a gratis donation of nifurtimox through an agreement with Bayer to match the gratis donation of eflornithine by Sanofi-Aventis. Influenced by the impact of the kit for eflornithine the World Health Organization also designed a new medical kit containing the combination in an easily accessible form and facilitated the training of key staff in disease endemic countries (DECs) to implement the drug combination.

For the first-stage disease, fortunately, in spite of the drugs having been used for over 70 years and requiring parenteral administration, similar safety and efficacy concerns have not yet emerged. Unfortunately to treat the second stage of rhodesiense HAT, melarsoprol remains the only drug available. This article presents the relative rates of use of available drugs to treat the second stage of gambiense HAT which accounts for $99.9 \%$ of the second stage of HAT cases reported in 2010 (World Health Organization, unpublished data) and discusses about the financial and social consequences.

THE PROCESS IN FACILITATING THE ACCESS TO THE BEST TREATMENT

Eflornithine has been donated to the World Health Organization since 2001 following an agreement between the manufacturer and WHO and has been provided free of charge to DECs. Its better safety profile, when compared to melarsoprol, rendered it the best treatment, in principle, for second-stage gambiense HAT. However, its use as a first-line treatment was restricted to relatively wealthy nongovernmental organizations (NGOs) because even when available free of charge due to its cumbersome and logistically demanding administration regime it proved prohibitively burdensome. Therefore only the small fraction of cases detected by NGOs had access to eflornithine while most of the cases detected by NSSCPs continued to be treated with melarsoprol (Simarro et al. 2011). Only some NSSCPs could afford its use but in these cases its use was generally reserved for melarsoprol refractory cases. For these reasons during the period 2001-2006 only a modest rise in use of eflornithine was recorded; an average $12 \%$ of all second-stage gambiense HAT cases detected during the period were treated with eflornithine (Fig. 1). 


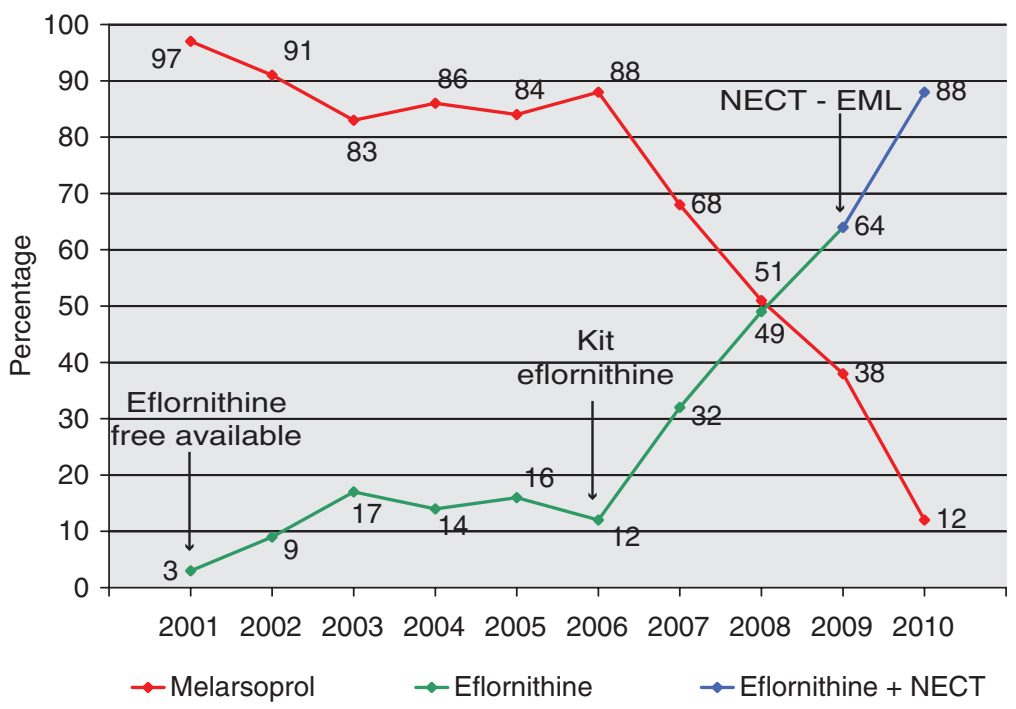

Fig. 1. Rate use of anti-trypanocidal drugs to treat second-stage gambiense HAT, 2001-2010. (NECT-EML: year when NECT was included in the Essential Medicines List of WHO).

In 2006, the World Health Organization was asked by representatives of the DECs for support with logistics and training, to allow NSSCPs to switch from melarsoprol to eflornithine as the first-line treatment. A training course was therefore organized for key staff from DECs to train in the administration of eflornithine and also to teach others how to administer the drug (Simarro et al. 2011). Moreover, a pre-packaged medical kit containing all the necessary drugs, infusion materials and accessories to administer 2 eflornithine treatments was designed. The kit's weight was $40 \mathrm{~kg}$, its volume $190 \mathrm{dm}^{3}$ and its cost $€ 1107$ including transport to health facilities in the HAT foci, making the cost of a single eflornithine treatment an estimated $€ 554$ (2010 prices).

In 2007, thanks to the enhanced number of trained technicians capable of administering treatment and the freely available medical kits, most NSSCPs switched to eflornithine as the first-line treatment in their HAT treatment policies. As a consequence, second-stage HAT gambiense cases treated with eflornithine increased by $167 \%$ (Fig. 1).

This new trend was reflected in a change in the distribution of recipients of eflornithine. From 2001 to 2006 the NGOs were the major recipients of distributed eflornithine, accounting for $89 \%$ of all used drug, while the NSSCPs only used 11\%. By contrast, over the period 2007-2009 the NSSCPs become the major consumers, using $65 \%$ of the total distributed eflornithine while the NGOs accounted for $35 \%$ (Simarro et al. 2011).

The inclusion of NECT in the EML in 2009 was followed by rapid addition of the combination therapy in the treatment policy of the NSSCPs. Previous efforts to encourage the implementation of eflornithine created a network of staff already trained in the required difficult parenteral infusion and management of the drug. Since use of NECT is also substantially less complex than eflornithine monotherapy (14 eflornithine infusions are given twice a day over 7 days, with associated oral nifurtimox during 10 days - as opposed to 56 infusions given 4 times a day over 14 days), the widespread uptake of NECT has been rapid. The World Health Organization continues to provide, in a new medical kit format, all the necessary drugs, infusion materials and accessories required to administer NECT. The reduced dosing of eflornithine means that the new medical kit for NECT, with the same volume as the eflornithine kit, now includes sufficient material for 4 treatments, weight $38 \mathrm{~kg}$ and costs $€ 1152$. The cost of 1 NECT treatment is therefore estimated at $€ 288$ (2010 prices) including transport to health facilities in the HAT foci.

As a result, in 2010, the use of melarsoprol has fallen to a historical low of just $12 \%$ of cases treated due to a larger use of melarsoprol alternative drugs (Fig. 1). In 2010 eflornithine monotherapy still accounted for $29 \%$ of treatments whereas NECT was the therapeutic regime of choice, used to treat $59 \%$ of all second-stage gambiense HAT cases reported that year.

THE FINANCIAL BURDEN OF ACCESS TO THE BEST TREATMENT

Historically, treatment of second-stage HAT has been considered a paradigm of dangerous drug usage when melarsoprol was the only available drug. The $5 \%$ fatality rate associated with adverse events was considered as the 'price to pay' to avoid the 100\% death rate from infection. The cost of ancillary drugs in the case of reactive encephalopathy during the use of melarsoprol was estimated as 20-50 USD (Robays et al. 2008). Ethically it is of paramount importance that people affected by HAT can access the best 


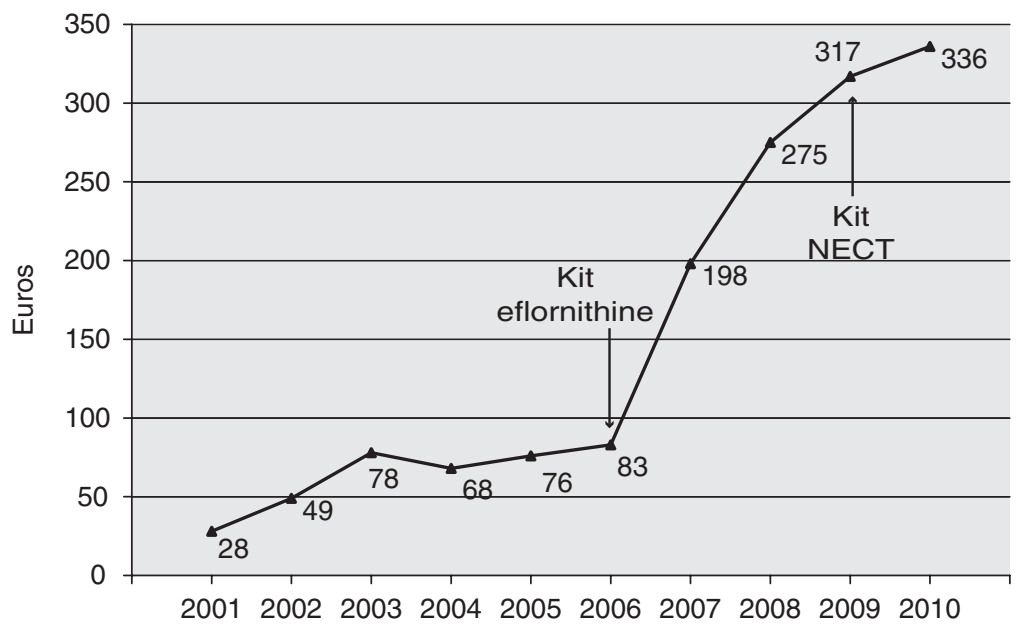

Fig. 2. Average cost to treat a second-stage gambiense HAT patient, 2001-2010.

treatment. However, fulfilling this prerogative carries financial consequences.

In 2001, 16614 second stages of gambiense HAT were reported (World Health Organization, unpublished data); $97 \%$ of them were treated with melarsoprol, the most toxic but least expensive drug available (Fig. 1). The average cost to treat 1 second-stage gambiense HAT patient was $€ 28$ (Fig. 2). Over the period 2001-2009 the use of less toxic but more expensive eflornithine increased 21 -fold from $3 \%$ to $64 \%$ (Fig. 1). Consequently, the average of cost of treating 1 second-stage gambiense HAT case over this period increased 11 times from $€ 28$ to $€ 317$ (Fig. 2).

In 2010, despite the implementation of NECT which is almost half the cost of the treatment with eflornithine (€288 versus $€ 554$ ), the average cost per second-stage gambiense HAT case treated has continued to rise reaching $€ 336$ (Fig. 2). Given that NECT is significantly cheaper than eflornithine monotherapy this appears surprising at first. However, the improved rate of uptake of NEC'T and consequent reduction in use of the far cheaper melarsoprol explains why (Fig. 1). This increased cost has had an impact on the distribution of the World Health Organization resources, made available mainly by Sanofi-Aventis, to support HAT control. From 2001 to 2005 , $€ 4386000$ (26\% of the resources) where dedicated to the access to treatment and 64245 patients were treated. From 2006 to 2010, €6 712300 (60\% of the resources) were allocated for access to the best treatment and 28064 patients were treated.

The shift from melarsoprol to eflornithine (in monotherapy and then as part of the NECT combination) has resulted in much safer treatment. Clearly, issues other than the simple cost per patient should influence policy making. For example, in 2001 the number of deaths related to treatment toxicity was estimated at 830 (5\% of cases treated) while in 2010 the number of deaths reported to be related to drug toxicity was $32(0.9 \%$ of cases treated). To date, health economists have not attempted to estimate the financial value of a human life, nor the relative value of the life of a sleeping sickness victim compared to others. Ethically, such calculations represent a huge challenge.

\section{DISCUSSION}

The development and implementation of NECT for HAT therapy has been proposed to carry 4 advantages over eflornithine monotherapy: (i) the combination is easier to administer and this has to lead to a wider use and consequent decrease in patients exposed to toxic melarsoprol. (ii) the combination is less expensive including anti-trypanosome and ancillary drugs, reducing the cost of the treatment compared with eflornithine monotherapy (iii) the combination is also considered to be less expensive for the patient due to the short time required in hospital reducing for the patient and the attendant the time out of the family environment and (iv) use in combination should diminish the risk of emergence of resistance to eflornithine. This latter point will only become clear in time. After 1 year of implementation of NEC'T the first proposed advantage has been demonstrated to be true. In 2010 we witnessed the lowest rate of melarsoprol use in second-stage gambiense HAT since records began. The second proposed advantage, however, appears to be confounded by an epidemiological and strategic detail that escaped initial prediction. Although the individual per-patient cost is $48 \%$ lower when using the combination NECT instead of eflornithine monotherapy, the average cost for treating second-stage gambiense HAT cases over the whole continent continued to increase by $6 \%$. This has led to the highest average cost per second-stage gambiense HAT ever. This apparent paradox is due to the ethical decision to use the safest but substantially more expensive alternative therapy NECT as a firstline treatment. 
National programmes have been able to select quality of treatment more than cost-effectiveness based on purely economical aspects. The added value in terms of decreased numbers of HAT patients exposed to and dying from melarsoprol toxicity, together with the lowering of adverse effects linked to treatment, and subsequent decrease in satellite treatment costs associated with those side-effects is great. NECT receives much better patient compliance and fewer patients abscond. Furthermore, health staff also suffer less stress than when administering melarsoprol. Unfortunately, it is difficult to attach a price to these benefits.

In spite of NECT's superiority in terms of safety, it is also important to remember that it is still far from ideal as a therapy. It still has to be administered by infusion over a protracted period, carries toxicity risks mainly fever, seizures and infections (Priotto et al. 2009). It is logistically difficult to deliver to and to manage in the settings in which it is required due to its parenteral administration and its volume. Above all, however, the logistical and financial burden that the therapy imposes on HAT control programmes will become a major issue and might render longterm application unsustainable. These two major negative factors call for continuing research to overcome these obstacles providing not only new safer and more effective drugs but also drugs which are simple to administer and cheaper when compared to current drugs.

\section{ACKNOWLEDGEMENT}

We thank MSF-Logistics for its collaboration in storing and dispatching drugs.

\section{REFERENCES}

Balasegaram, M., Harris, S., Checchi, F., Ghorashian, S., Hamel, C. and Karunakara, U. (2006). Melarsoprol versus eflornithine for treating late-stage Gambian trypanosomiasis in the Republic of the Congo. Bulletin of the World Health Organization 84, 783-791.

Bisser, S., N'siesi, F.X., Lejon, V., Preux, P.M., Van Nieuwenhove, S., Miaka Mia Bilenge, C. and Buscher, P. (2007) Equivalence trial of melarsoprol and nifurtimox monotherapy and combination therapy for the treatment of second-stage Trypanosoma brucei gambiense sleeping sickness. Fournal of Infectious Diseases 195, 322-329.

Blum, J., Nkunku, S. and Burri, C. (2001). Clinical description of encephalopathic syndromes and risk factors for their occurrence and outcome during melarsoprol treatment of human African trypanosomiasis. Tropical Medicine and International Health 6, 390-400.
Burri, C. (2010). Chemotherapy against human African trypanosomiasis: Is there a road of success ? Parasitology 137, 1987-1994.

Chappuis, F., Udayraj, N., Stietenroth, K., Meussen, A. and Bovier, P. A. (2005). Eflornithine is safer than melarsoprol for the treatment of second-stage Trypanosoma brucei gambiense human African trypanosomiasis. Clinical Infectious Diseases 41, 748-751.

Checchi, F., Piola, P., Ayikoru, H., Thomas, F., Legros, D. and Priotto, G. (2007). Nifurtimox plus eflornithine for late-stage sleeping sickness in Uganda: a case series. PLoS Neglected Tropical Diseases 1, e64. Janssens, P. G. and De Muynck, A. (1977). Clinical trials with "nifurtimox" in African trypanosomiasis. Annales Societé Belge Médicine Tropicale 57, 475-480.

Legros, D., Evans, S., Maiso, F., Eyaru, J. C. K. and Mbulamberi, D. (1999). Risk factors for treatment failure after melarsoprol for Trypanosoma brucei gambiense trypanosomiasis in Uganda. Transactions of the Royal Society of Tropical Medicine and Hygiene 93, 439-442.

Moens, F., De Wilde, M. and Ngato, K. (1984). Clinical trial of nifurtimox in human African trypanosomiasis. Annales Societé Belge Médicine Tropicale 64, 37-43.

Moore, A.C. (2005). Prospects for improving African trypanosomiasis chemotherapy. The Fournal of Infectious Diseases 191, 1793-1795.

Pepin, J., Milord, F., Meurice, F., Ethier, L., Loko, L. and Mpia, B. (1992). High-dose nifurtimox for arseno-resistant Trypanosoma brucei gambiense sleeping sickness: an open trial in central Zaire. Transactions of the Royal Society of Tropical Medicine and Hygiene 86, 254-256.

Priotto, G., Fogg, C., Balasegaram, M., Erphas, O., Louga, A., Checchi, F., Ghabri, S. and Piola, P. (2006). Three drug combinations for late-stage Trypanosoma brucei gambiense sleeping sickness: A randomized clinical trial in Uganda. PLoS Clinical Trials 1, e39: 31-38.

Priotto, G., Kasparian, S., Mutombo, W., Ngouama, D., Ghorashian, S., Arnold, U., Ghabri, S., Baudin, E., Buard, V., Kazadi-Kyanza, S., Ilunga, M., Mutangala, W., Pohlig, G., Schmid, C., Karunakara, U., Torreele, E. and Kande, V. (2009). Nifurtimox-eflornithine combination therapy for second-stage African Trypanosoma brucei gambiense trypanosomiasis: a multicentre, randomised, phase III, non-inferiority trial. Lancet 374, 56-64.

Priotto, G., Pinoges, L., Badi Fursa, I., Burke, B., Nicolay, N., Grillet, G., Hewison, C. and Balasegaram, M. (2008). Safety and effectiveness of first line eflornithine for Trypanosoma brucei gambiense sleeping sickness in Sudan. British Medical Fournal 336, 705-708.

Robays, J., Raguenaud, M. E., Josenando, T. and Boelaert, M. (2008). Eflornithine is a cost-effective alternative to melarsoprol for the treatment of second-stage human West African trypanosomiasis in Caxito, Angola. Tropical Medicine and International Health 13, 265-271.

Seixas, J. (2004). Investigations on the encephalopathic syndrome during melarsoprol treatment of human African trypanosomiasis. Ph.D. thesis, Instituto de Higiene e Medicina Tropical, Universidade Nova de Lisboa. Simarro, P.P., Diarra, A., Ruiz Postigo, J. A., Franco, J. R. and Jannin, J. G. (2011). The Human African Trypanosomiasis Control and Surveillance Programme of the World Health Organization 2000-2009: The Way Forward. PLoS Neglected Tropical Diseases 5, e1007. doi: 10.1371/ journal.pntd.0001007.

Van Nieuwenhove, S. (2000). Gambiense sleeping sickness: re-emerging and soon untreatable? Bulletin of the World Health Organization 78, 1283.

Vincent, I. M., Creek, D., Watson, D. G., Kamleh, M. A., Woods, D. J., Pui EeWong, P.E., Burchmore, R. J.S. and Barrett, M.P. (2010). A Molecular Mechanism for Eflornithine Resistance in African Trypanosomes. PLoS Pathogens 6, e1001204. doi: 10.1371/journal. ppat.1001204.

World Health Organization (2009). WHO model list of essential medicines, 16th list March 2009. World Health Organization, Geneva, Switzerland. http://www.who.int/medicines/publications/essential medicines/en/index.html. 